\title{
Fluctuations of Solar Wind Parameters During Polar Reversal
}

\author{
Iren Sobia, Bidhu S. S., Dickson Benjamin \\ Department of Physics, Nesamony Memorial Christian College, Marthandam, Tamil Nadu, India
}

Email address:

irenbsobia@gmail.com (I. Sobia), bidhuss@gmail.com (Bidhu S. S), dicksonbenjamin@gmail.com (D. Benjamin)

To cite this article:

Iren Sobia, Bidhu S. S., Dickson Benjamin. Fluctuations of Solar Wind Parameters During Polar Reversal. American Journal of Astronomy and Astrophysics. Vol. 3, No. 3, 2015, pp. 56-62. doi: 10.11648/j.ajaa.20150303.15

\begin{abstract}
Around the time of polarity reversal in 2000 - 2001, the solar wind plasma parameters and interplanetary magnetic field fluctuations are studied for an interval of time that corresponds to Ulysses in-situ measurements of high-latitude heliospheric magnetic field. This study has been done by investigating solar wind speed, density, temperature and solar magnetic field using Ulysses magnetometer and SWOOPS instrument. The sun's magnetic field reverses polarity approximately in every 11 year and it creates a peak in each solar cycle. This study gives a brief understanding of solar wind parameters in the heliosphere during polar reversal in solar cycle 23. The solar magnetic field completely reorganizes during the polar reversal phase, hence the distribution of solar wind parameters changes accordingly. By studying the variation of solar wind parameters, it is possible to understand the polar reversal phenomenon. The photospheric polarity reversal is completed in more active Northern Hemisphere in late 2000 and then in the Southern Hemisphere in 2001. The reversal of the magnetic field at the solar wind source surface is inferred to have occurred between late 2000 and 2001, with the most likely time of reversal being early within that period.
\end{abstract}

Keywords: Heliosphere, Interplanetary Magnetic Field, Solar Wind

\section{Introduction}

The solar wind is magnetized plasma throughout the solar system. In which the magnetic and plasma pressures are comparable. The high variability of the solar wind in space and time reflects the underlying coronal structures [1]. The high electrical conductivity of plasma, forces the part of the solar magnetic field to move out wards, as if it were frozen into the plasma. This field called interplanetary magnetic field (IMF). It is embedded in the solar wind and carries while it passes the Earth. It is highly determined by the solar wind couples with the remote terrestrial atmosphere. Although the solar wind does not penetrate down to the ground, it is highly significant in geospace and some of the most remarkable behavior is directly attributable to the variations of the solar wind and its magnetic field.

The Sun has a steady but highly variable supersonic outflow of charged particles, magnetic field, and energy called the solar wind. The solar wind is bimodal, fast or slow with velocities ranging from $400-900 \mathrm{~km} \mathrm{sec}^{-1}$. At $1 \mathrm{AU}$, normal densities for the slow wind are $8 \mathrm{~cm}^{-3}$ [2], with a proton temperature of $1 \times 10^{5} \mathrm{~K}$ and a speed of $400 \mathrm{~km} \mathrm{sec}^{-1}$. In the fast solar wind the density drops to around $2.5 \mathrm{~cm}^{-3}$ and with a mean speed of $770 \mathrm{~km} \mathrm{sec}^{-1}$ [2]. The slow solar wind is associated with field lines near closed magnetic regions that open up and allow an outflow of material for a short time $[3,4]$. The solar wind varies with the solar cycle. Coronal holes occur at all latitudes and are smaller. Therefore, the fast wind is not restricted to the polar area [5]. Similarly, slow solar wind sources extend to higher latitudes.

One of the striking features in the solar activity cycle is the reversal of polarity of the polar magnetic field. The solar dipole magnetic field reverses its polarity around the maximum phase of the solar activity. The characteristics of the polar reversal play a significant role in determining the coronal and interplanetary magnetic fields. During the reversal phase the solar surface is occupied with mixed polarities. The polarities often form closed magnetic loops, found to spread over wide coronal surface, accompanying small coronal holes that forms ambient solar wind from the surface of Sun. The polarity reversal at the poles starts after sunspot maximum. The solar wind that emerges during the polarity reversal is found to have slow solar speed with high concentrations of proton particle.

The solar activity and its influence is a consequence of the 
existence of the sun's magnetic field. The structure of the heliosphere is heavily dominated by this field, the solar wind and the rotation of the Sun. Due to the almost infinite conductivity of the plasma, the magnetic field is frozen into the solar wind. In this way, the shape of the magnetic field lines will be determined by the plasma flow velocity and the Sun's rotation. The fact that the Sun's magnetic field is not only constantly expanding, but it is slightly offset from the Sun's axis of rotation is also considered. One of the important consequences of this model is that field lines connect directly from low to high heliospheric latitudes, allowing for example the transport of energetic particles from low to high latitudes without the need for cross-field diffusion.

\section{Instrumentation}

The HMF polarity distributions were derived from data returned by Ulysses's magnetometer [6] and SWOOPS instrument [7]. The joint NASA/ESA Ulysses mission has provided the first direct, in-situ measurement of high latitude, slow as well as dense solar wind in the interplanetary medium. Ulysses orbits two times around the Sun, which covers the declaining phase of solar cycle 22 and ascending phase of solar cycle 23 . The observations from two full orbits provide the complete information about the spatial structure of solar wind with respect to the solar phase. The data from SWOOPS (Solar Wind Observations over the Poles of the Sun) and VHM (Vector Helium Magnetometer) Ulysses experiments are utilized for carrying out this work. The solar magnetic parameters and solar wind plasma parameters are used for the analysis. The parameters are scaled to $1 \mathrm{AU}$ and averaged over latitude.

\section{Data Analysis and Observation}

Comparison of observations from Ulysses's first two orbits demonstrated, that the structure of the solar wind varies dramatically over the solar cycle. McComas et al., [8,9] explained that the declining phase of solar cycle 22 and through solar minimum ie., in the Ulysses' first orbit, the solar wind displayed a simple bimodal structure with persistently fast tenuous and uniform solar wind at high heliolatitudes and slower, more variable, and highly structured wind at low latitudes. In the mid- latitudes Ulysses observed a large, stable co-rotating interaction region (CIR) [10] formed by the interaction between slow solar wind and faster coronal hole wind overtaking it. Around solar minimum, the band of solar wind variability was narrow, and confined to $\sim 30^{\circ}$ to $\sim 20^{\circ} \mathrm{N}$ latitudes indicative of small dipole tilt angles and confinement of coronal helmet streamers to low latitudes at these times $[8,11]$.

During the rise to and throughout solar maximum in solar cycle 23 ie., in the Ulysses's second orbit the global structure of the solar wind was completely different. At these times the solar wind was highly variable with flows arising from a mixture of sources including streamers, coronal mass ejections (CMEs), small coronal holes, and active regions [12] at all heliolatitudes [13]. Throughout this interval the two-sector magnetic structure (inward and outward spiral fields) persisted, but around maximum the apparent dipole axis was oriented nearly perpendicular to the rotation axis as the Sun reversed its magnetic polarity.

\section{Characteristics of Solar Wind Parameters}

From Figure 1, it shows four panels of radial interplanetary magnetic field, proton density, temperature and solar wind speed. The parameters are drawn by taking running average of Carrington rotations. The variation of solar wind parameters with respect to radial magnetic field is displayed. The first panel of the Figure 1 shows the polarity change of interplanetary magnetic field of radial component. The Sun's global magnetic dipole field structure dominates during the minimum phase of the Sun. In the first panel of the Figure 1, the dipole structure keeps the equatorial corona tied to the Sun leaving the other regions magnetically open. During the maximum phase, in addition to the global dipole field, the toroidal fields (sunspots) spread over the mid latitude and equatorial regions of the surface of Sun. This makes the Sun a multipolar one and breaks its temporal and spatial homogeneity. The Ulysses's measurement of IMF during solar minimum is consistent to our expectation. Equatorial regions push more flux in to the interplanetary medium than the high latitudinal regions.

During maximum solar activity, closed or mixed magnetic structures and slow wind dominate. In the epoch of minimum activity, the magnetic structures are mostly of an open type and generate fast solar wind streams [14]. Near the solar maximum, the polar fields are weak and in the process of reversing, while the fields in the active regions which determine the resultant equatorial dipole are numerous and strong [15]. Ulysses observed low latitude streamers near heliospheric current sheet during minimum phase. The streamers show its presence in higher latitudes, as the Sun gains magnetic multipoles in the maximum phase. During minimum phase, polarity fluctuations are always confined to ecliptic region, because of the presence Sun spots near the equatorial plane. During maximum phase, most of the solar surface is occupied by regions of magnetic multi poles, except the narrow polar region.

The second panel of Figure 1 shows proton particle variations, which shows variations alike the radial IMF variations. The flux density for high speed is always smaller and connected to open field regions. The closed field regions are associated with solar wind and slow speed. Ulysses observation of slow wind is associated with relatively high proton density. The third and forth panel of Figure 1 shows the proton temperature and the solar wind velocity. The variations of solar wind temperature and solar wind speed are dependent with each other showing its strong mutual inter dependence. In general, proton density is greater for slower solar wind. The magnetic sector boundary crossings are identified as regions 
of increased number of protons [16]. The proton number observed by Ulysses has been maximum in the equatorial regions, where the solar wind streams' velocity is extremely very low from figure 1 . Here, the proton emission is in fact independent of any latitudinal structure [17]. The minimum density of solar wind particle near ecliptic plane is observed during magnetic field polarity reversal in the polar region [18].

The third panel of Figure 1 shows that, at the equatorial regions the solar wind temperature and wind speed is of minimum value. The solar cycle variation of temperature in the outer heliosphere is much smaller than that observed at 1 AU. The latitudinal dependence of the proton temperature decreases towards the current sheet [17]. The most important result obtained in this work is the presence of strong latitudinal gradient in solar wind temperature. The gradient is an extended phenomenon and does not appear to be associated with a particular phase of the solar cycle [19]. The latitude gradient remains almost unchanged over the course of solar maximum phase as observed by Ulysses in its second orbit around the Sun. The average solar wind temperature appeared to vary with solar cycle. It is lower near solar maximum and higher near solar minimum. This is not surprising, since similar behavior has previously been observed in $1 \mathrm{AU}$, but the solar cycle variation of temperature in the outer heliosphere is much smaller than that observed near 1 AU. The most striking behavior of our observation is the strong latitudinal variation in solar wind temperature.

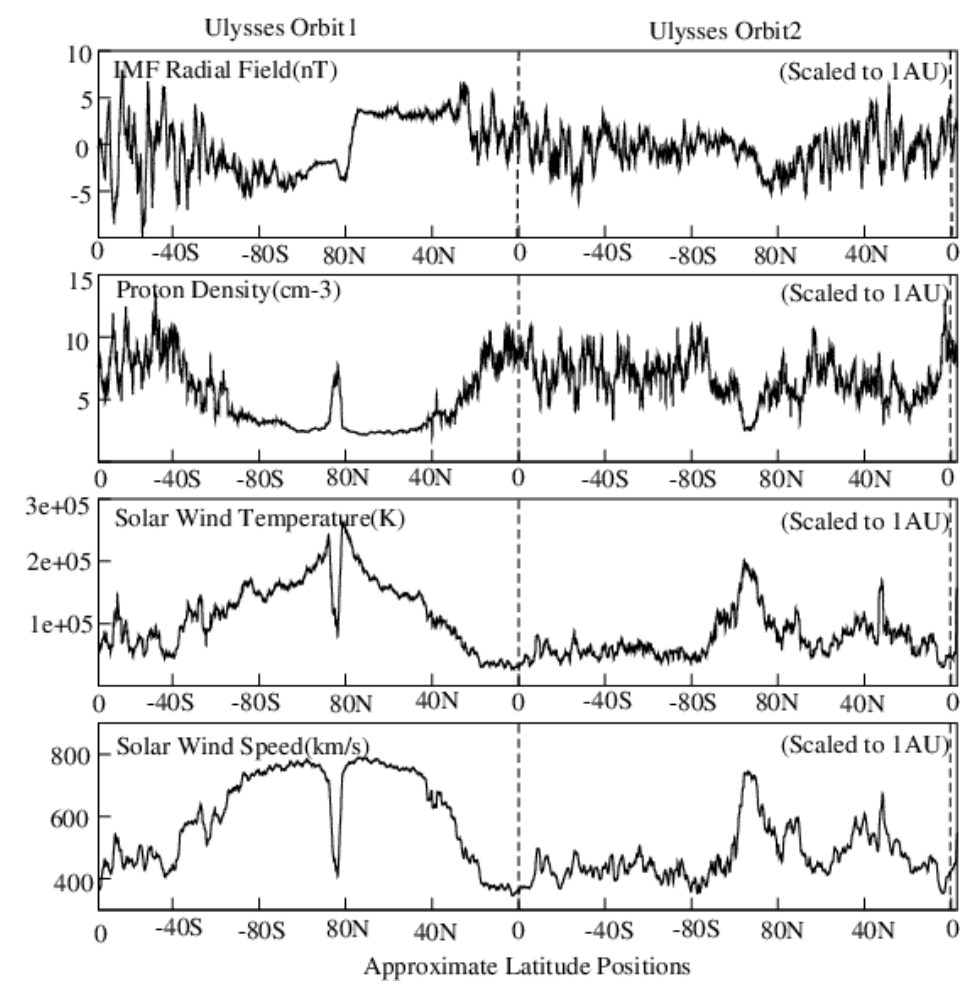

Figure 1. From top to bottom: Radial interplanetary magnetic field, Proton density, Proton temperature and Solar wind speed.

The solar wind temperature with respect to lattitude is exhibited in the Southern hemisphere. The temperature varies linearly; in Northern hemisphere variations are much more. The latitude gradient remains almost unchanged over the course of solar maximum phase, as observed by Ulysses in its second orbit around the Sun. The average solar wind temperature appeared to vary with solar cycle.

The slow solar wind $(400 \mathrm{~km} / \mathrm{s})$, is the characteristic nature of polar reversal commencement of the middle polar reversal. While this, the fast wind dominates $800 \mathrm{~km} / \mathrm{s}$. Solar wind structure in the southern hemisphere, comprises a mixture of solar wind streams from variety of sources, both in the low and high latitudes. [20] Found that, very low speed regions observed in the solar maximum are associated with solar active regions. The general decreases in the solar wind velocity are found to be common at solar maximum. This seems to be associated with the Sun's polar field reversals.
The corpuscular emission from the Sun appears to be decelerated to considerable degree in spite of high sporadic solar activity [21], during these periods. Kojima and Kakinuma [22] have shown that low speed distributes widen up to higher latitudes during solar activity maximum.

As Ulysses ascended in latitude during the maximum phase, due to the increased solar activity, the slow interactive wind gradually expanded to all heliolatitudes and the fast high latitude wind disappeared [23]. It is firmly proved that the fast solar wind originates exclusively from coronal holes. The solar wind from the polar coronal holes expands super radially, producing high speed streams near the solar equator [24]. In addition to the latitudinal variations in the solar wind plasma parameters, asymmetries have also been observed between the northern and the southern hemispheres during the two phases of solar activity [25]. Large scale compressional structures generated between the high and low speed streams dominate in 
the low latitude solar wind and the constant smooth high speed flow occupies the polar coronal hole [26]. The high speed solar wind streams are observed when the polar coronal hole extends to equator. This normally happens in the descending phase of the solar cycle. Also, high speed streams are observed during periods of slight extension of coronal hole towards the equator followed by axial tilt of the Sun and slight deviation of the spacecraft from ecliptic position [27]. The recurrent fast solar wind streams dominate in mid and low latitudes when the Sunspots are minimum in mid and low latitudes [28].

The fourth panel of Figure 1 shows the flow of solar wind measured by Ulysses during the maximum phase of solar activity. The fast streams, prevailing at higher latitudes during minimum, disappear almost entirely during solar maximum, and the wind becomes slow and highly structured in space and variable in time. But for few sharp peaks in the ecliptic region, the solar wind is very slow and passive in the southern hemisphere. Unlike in the southern hemisphere, the solar wind is very erratic in the northern hemisphere and the solar wind speed is substantially enhanced. The Ulysses observed a polar coronal hole with smaller area compared to that observed during the minimum phase. Maximum phase is coincided with the solar polarity reversal. Well, before the reversal phase, the solar wind is found to be comparatively slower and smoother. A limited number of coronal transient are observed during the first polar orbit of Ulysses. Since the occurrence rate of solar transients follows the solar activity cycle, Ulysses observed more solar transients during the maximum than the solar minimum. The enhanced solar transient during maximum phase is the main reason for erratic variations of solar wind speed. Ulysses observations show that slow and mixed solar wind extends to higher latitudes at solar maximum.

\section{The Magnetic Field Polarity Distribution}

The solar wind basically flows with two distinct streams, high speed and low speed with different physical conditions. The coronal hole, which is an open field and a low density region, is an established region of the high speed wind. The solar wind from the structured corona with heliospheric current sheet (HSC) has been known for its low speed streams. The low speed solar wind near IMF polarity reversal has been observed by Nerney [34]. The slow solar wind is related to closed solar magnetic lines and the HCS [29]. The spacecraft's first fast latitude scan, during which Ulysses travelled from high southern to high northern latitudes in less than 11 months, took place near a minimum in the solar cycle. Solar magnetic polarity reversal took place when the spacecraft recently repeated the scan near solar maximum. The open magnetic field lines originating in regions of opposite magnetic polarity are separated by the heliospheric current sheet (HCS), which is explained by [31]. Near the solar maximum, as the dipole begins to reverse orientation, the HCS is expected to extend over an ever-increasing heliolatitude range.

At solar maximum slow wind dominates in the beginning of Ulysses second orbit, which may be due to the reformation of wide coronal holes in the maximum phase [35]. Ohmi et al., [36] have observed low speed solar wind for a short period near the poles during the solar maximum phase. The evolution of slow speed solar wind and its origin have been investigated and compared with the solar magnetic field. Miyake et al., [27] compared the solar wind structure with IMF polarity and found, high speed streams usually have a single polarity. The magnetic field becomes multi-polar towards maximum activity.
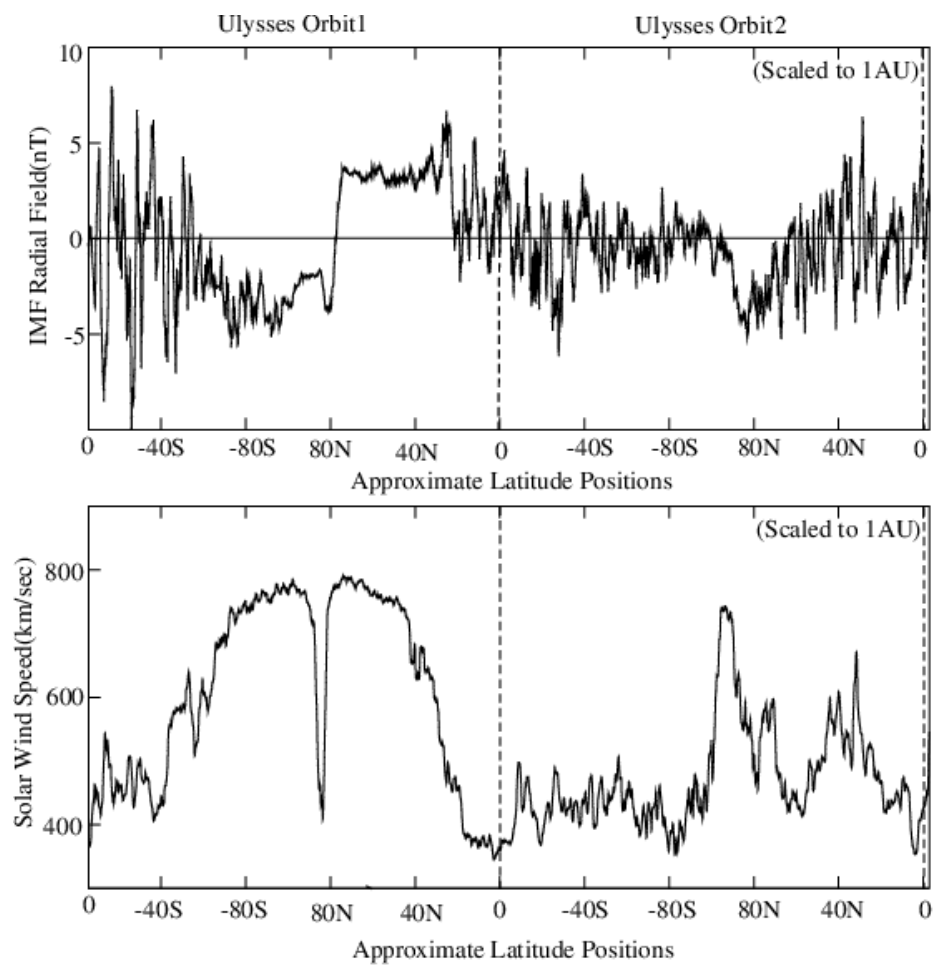

Figure 2. Plot showing Solar Wind Speed and IMF radial field. 
The HCS was confined to low heliolatitudes, during the majority of Ulysses's first polar orbit. Data returned by Ulysses during 2000 - 2001 have provided the first in situ measurements of high-latitude heliospheric magnetic fields around the time of polarity reversal, when the HCS extended over its maximum heliolatitude range. The Ulysses data may thus greatly aid to our understanding of the polarity reversal process. Ulysses in its second rotation observed the existence of multi-poles in the southern hemisphere, even in high latitude.

The decrease solar wind speed near solar maximum have been studied in detail and Figure 2 clearly shows that the slowing down of solar wind is associated with solar active regions of multi-poles. The figure points out that magnetic field with multi polarity regions with equal strength in polarity (positive - negative) highly restrict the flow of solar wind. The origin of low speed wind flow has been not thoroughly explained, unlike the fast speed stream which emanates from the open field regions of coronal holes. Normally the low speed flow is confined to low latitude regions. Moreover the magnetic field components of radial field (global solar field) and the normal field aligned to strengthen the magnetic structure in that region. That the IMF polarity reversal was completed at the south pole well after July 2000 has been confirmed by Ulysses magnetometer measurements, which showed predominantly negative polarities near latitude $80^{\circ} \mathrm{S}$ during November 2000 [37].

\section{The Polarity Reversal in Ulysses Second Orbit}

The polarities of the solar magnetic field are shown in Figure 3. In the solar maximum period, increase complexity in the sector structure detected at Ulysses, coincident with the increase in solar activity [29] described the observations to mid of 2000 and [33] summarized observations at Ulysses to late 2000, at high southern heliolatitudes. The Southern Hemisphere not being dominated by a single polarity act as a mixture of the two polarities, whereas the HCS extends to ever higher heliolatitudes, with the approach of solar maximum. During each solar rotation, as two polarities were detected except for the very last rotation prior to maximum southern heliolatitude [33], and this indicates that Ulysses briefly moved to higher latitudes than the maximum HCS latitude. It can be stated that magnetic polarity reversal had not occurred at last time of the 2000 , at least in the polarity distribution at the solar wind source surface, if the HCS during this time was a single, coherent structure without significant warps. A small warp in the HCS would have been sufficient to produce the observations described while most of the sheet had actually tipped past the south pole, although it is almost certain that the HCS inclination was near its maximum. Balogh et al., [30] explained by, the two-sector structure observed during this period was consistent with a single HCS tilted at high inclination.

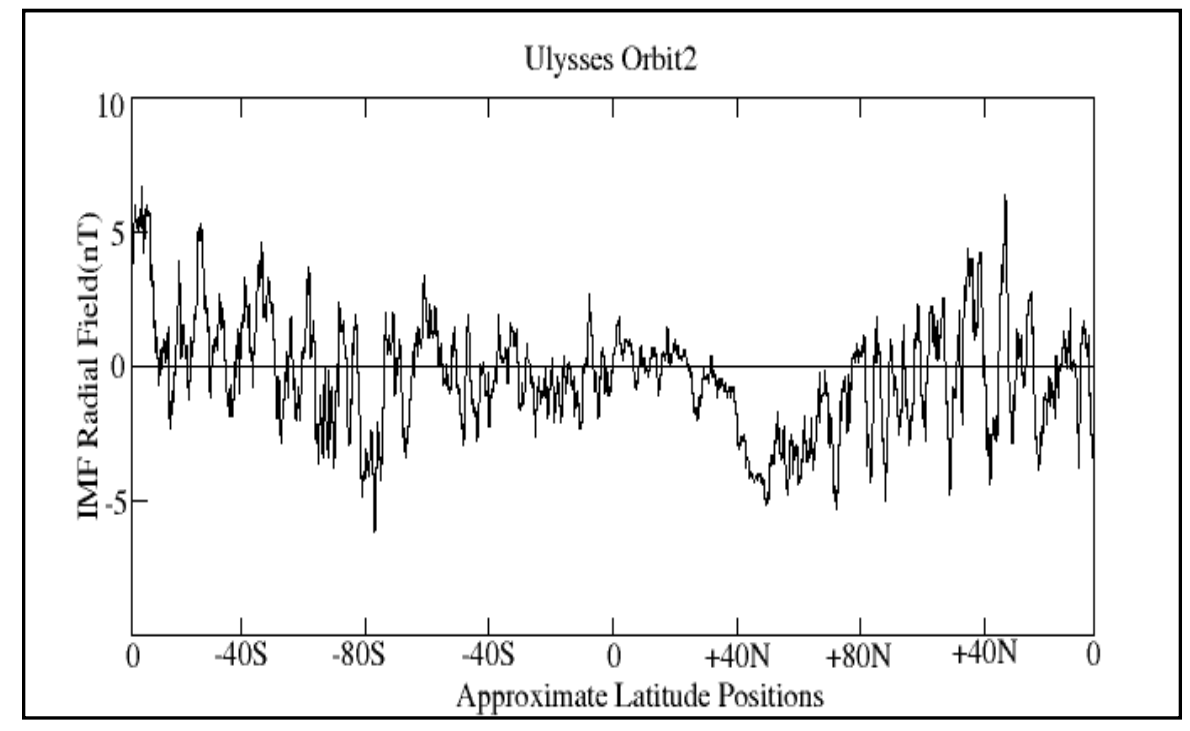

Figure 3. Plot showing the polarity reversal.

An initial overview of the HMF polarity at Ulysses around solar maximum was presented by [31] and this period is represented in the Figure 3. Between this mission phase and solar minimum fast latitude scan, a lot of obvious differences in the polarity distribution can be sorted out. Although the polarity distribution was probably evolving rapidly during this period, the fast pace of Ulysses's motion around its second perihelion of the HMF polarities during this key phase in the solar activity cycle. During almost the entire period, both polarities continued to be detected at the space craft, in approximately equal proportions with each solar rotation, until northern mid-heliolatitudes were reached. The northern polarity was the only one detected with the exception of brief reversals within transient structures, from the last clear HCS crossing was seen at Ulysses, at a heliolatitude of $\sim 68^{\circ} \mathrm{N}$, around middle of the 2001. That time onwards, as the new northern polarity was clearly dominant, this detection constrains the time of polarity reversal at the source surface to earlier than middle of the 2001.

A complex mixture of fast and intermediate speed flows, 
probably resulting from interactions between slow streams and faster flows from coronal holes and the interplanetary counterparts of coronal mass ejection [32] are found to be seen in the Solar wind parameters during this period. The magnetic polarity distribution is, however, simple enough, with a two-sector structure seen in the $\sim 80^{\circ} \mathrm{S}$ to $\sim 40^{\circ} \mathrm{N}$ heliolatitude range. It can be stated confidently that the sector structure seen was a result of the solar magnetic dipole being tipped, such that its axis was near orthogonal to the Sun's rotation axis.

From the Figure 3, the polarity distribution, in which Ulysses has moved from high northern latitudes to $\sim 30^{\circ} \mathrm{N}$, is candidly seen. The sector structure when detected again, around the starting days of 2002, at a heliolatitude of $\sim 62^{\circ} \mathrm{N}$, the possible sector obtained was not clear, and it was two full solar rotations. Later, when the sector structure was detected unambiguously, at heliolatitude of $\sim 50^{\circ} \mathrm{N}$, the sector structure continues to be quite clearly seen, with the new northern polarity constituting most of the solar wind sampled, as expected by Jones and Balogh [31].

\section{Discussion and Conclusion}

Solar wind velocity and temperature are higher at high latitudes, while the density is lower, especially around coronal holes. The fast streams, prevailing at higher latitudes during minimum, disappear almost entirely during solar maximum, and the wind becomes slow and highly structured in space and variable in time. Ulysses observations show that slow and mixed solar wind extends to higher latitudes at solar maximum. The solar wind at low latitudes tends to be structured into alternating streams of high and low speed flows that rotate with the Sun. The Ulysses measurement of interplanetary field during minimum is consistent to our expectation. Ulysses observed highly variable and almost equal IMF in all heliolatitudes during its second orbit. The average solar wind temperature is lower near solar maximum and higher near solar minimum. The most striking result of our observations is the strong latitudinal gradient in solar wind temperature and the latitudinal gradient remains almost unchanged over the course of solar maximum.

The data presented here represents first direct measurements of the solar polar magnetic fields at solar maximum. From this observation as expected, the polarity distribution was much more complex than that near solar minimum. The polarity distribution detected by Ulysses indicates that the magnetic reversal in the solar wind source surface occurred between November 2000 and August 2001, and is likely to have been early within that time range. During the solar maximum phase, Ulysses observed negative polarity in the southern hemisphere and once again it recorded negative polarity in the northern hemisphere while moving quickly from the southern hemisphere to northern hemisphere. The Sun behaved like a monopole and this effect is carried in to the interplanetary magnetic field. This change is attributed to the phenomenon of polarity reversal. The overall field is minimum around poles and maximum around the mid latitudes, while the sunspot field dominates during the maximum phase. At solar maximum the slow wind dominates in the beginning of Ulysses second orbit, which may be due to the reformation of wide coronal holes in the maximum phase.

\section{Acknowledgements}

We thank all the wonderful men and women who have made the Ulysses program such an outstanding success. The use of data of the plasma analyser (principal investigator D. J. McComas, Southwest Research Institute, San Antonio, Texas, USA) and of the magnetometers (principal investigator A. Balogh, The Blackett Laboratory, Imperial College, London, UK) aboard the Ulysses spacecraft is gratefully acknowledged. The data have been made available through the World Data Center A for Rockets and Satellites (NASA/GSFC, Greenbelt, Maryland, USA). The present work has been supported by Nesamony Memorial Christian College, Marthandam.

\section{References}

[1] M. B. Kallenrode, 2 ed, Springer, Germany, (2001).

[2] T.I. Gombosi, Physics of the Space Environment, ISBN 052159264X, Cambridge University Press, (1999).

[3] Y. M. Wang and N. R. Sheeley, ApJ 355 , 726, (1990).

[4] L. A. Fisk, Journal of Geophysical Research (Space Physics) 108(A4), 7 (2003).

[5] R. Woo, and S.R. Habbal, Geophys Res Lett, 24, 1159, (1997).

[6] A. Balogh, T. J. Beek, R.J. Forsyth, P. C. Hedgecock, R.J. Marquedant, E. J. Smith, D. J. Southwood, and B. T. Tsurutani, Astron Astrophys Suppl Ser, 92, 221-236, (1992).

[7] S. J. Bame, D. J. McComas, B.L. Barraclough, J.L. Phillips, K. J. Sofaly, J. C. Chavez, B.E. Goldstein, and R. K. Sakurai, Astron Astrophys Suppl Ser, 92, 237-265, (1992).

[8] D. J. McComas, Geophys Res Lett, 25, 1-4, (1998a).

[9] D. J. McComas, Geophys Res Lett, 105, 10,419, (2000).

[10] J.T. Gosling, Annu Rev Astron Astrophys, 34, 35 - 73, (1996).

[11] J.T. Gosling, S.J. Bame,W.C. Feldman, D. J. McComas, P. Riley, B.E. Goldstein, and M. Neugebauer, Geophys Res Lett, 24, 309-312, (1997).

[12] M. Neugebauer, P. C. Liewer, E. J. Smith, R. M. Skoug, and T. H. Zurbuchen, J Geophys Res, 107(A12), 1488, (2002).

[13] D. J. McComas, AIP Conf Proc, 679, 33, (2003).

[14] N. A. Lotova, V. N. Obridko, K. V. Vladdimirsk, M. K. Bird, and P. Janardhan, Solar Phys, 205, 149-163, (2002).

[15] E. J. Smith, J. Geophys. Res., 106, 15, 819-831, (2001).

[16] J. T. Steinberg, and A. Lazarus, Geophys Res Lett, 23, 1183 $-1186,(1996)$.

[17] R. Bruno, Schwann, and F. Mariani, Solar Phys, 104, 431-445, (1986).

[18] K.A. Kovalenko, Planet Space Sci, 36, 1343-1358, (1988). 
[19] P. R. Gazis, A. Barnes, J. D. Mihalov, and A. J. Lazarus , J. Geophys. Res., 99, 6561-6573, (1994).

[20] H. Watananbe, M. Kojima, Y. Kouzuka and Y. Yamuchi, AIP Conf. Proc, 382, 117 (1996).

[21] Prigancov, Adv Space Res, 11, 41-46, (1991).

[22] Kojima M, and Kakinuma T , J. Geophys. Res., 92, 7269 7279, (1987).

[23] E. J. Smith, and R. G. Marsden, Geophys. Res. Lett., 30, ULY1 $1-4,(2003)$.

[24] R. Woo, and S. R. Habbal , J. Geophys. Res., 105, 12, 667 - 674, (2000).

[25] D. J. McComas, and J. T. Gosling, Geo phys. Res. Lett., 27, 2437-2430, (2000).

[26] D. J. McComas, B. Barraclough, J. Gosling, C. Hammond, J. Phillips, M. Neugebauer, A. Balogh, and R. Forsyth, Geophys. Res. Lett., 105, 19, 893 - 902, (1995a).

[27] W. Miyake, K. Kobayashi, K. I. Oyama, T. Mukai, T. Abe, T. Terasama, K. Yu-moto, T. Saito, K. Hira, A. J. Lazarus, and A. D. Johnstone, Planet. Space Sci, 12, 1329-1342, (1988).
[28] H. H. Sargent, J. Geophys. Res., 90, 1425-1428, (1985).

[29] N. R. Sheeley, J. W. Harvey, and W. C. Feldman, Solar Phys, 49, 271, (1976).

[30] A. Balogh and E. J. Smith, Space Sci Rev, 97, 147- 160, (2001).

[31] G. H. Jones and A. Balogh, Ann Geo, 21, 1377 - 1382, (2003).

[32] D. J. McComas, H. A. Elliott, J.T. Gosling, D.B. Reisenfeld, R. M. Skoug, B. E. Goldstein, M. Neugebauer, and A. Balogh, Geophys Res Lett, 29, 9, (2002).

[33] E. J. Smith, A. Balogh, R. J. Forsyth, and D. J.McComas, Geophys Res Lett, 28, 4159-4162, (2001).

[34] S. Nerney, and S. T. Suess, Astron J, 296, 259-267, (1985a).

[35] C. P. Pagel, and A. Balouh, J Geophys Res, 107, SSH6 1-4, (2002).

[36] T. Ohmi, M. Kojima, A. Yokobe, M. Tokumaru, and K. Fujiki, J. Geophys Res, 106, 24923 - 24936, (2001).

[37] P. Janardhan, K. Fujiki, M. Kojima, and M. Tokumaru, Proc. Of the ILWS Workshop, 132-139, (2006). 